\title{
PERFORATIONS COMPLICATING JEJUNAL DIVERTICULA: OUR EXPERIENCE
}

\author{
J. Ray, P.K. Singh, S. Kumar, S.K. Sahu ${ }^{凶}$, P.K. Sachan \\ Department of Surgery, Himalayan Institute of Medical Sciences
}

\begin{abstract}
PERFORATIONS COMPLICATING JEJUNAL DIVERTICULA: OUR EXPERIENCE (Abstract): Single jejunal diverticula are rare surgical entities. They sometimes present clinically due to complications, such as: perforation, bleeding, obstruction, etc. In this retrospective study we have included three cases of single jejunal diverticula complicated by perforation and subsequent peritonitis. All cases underwent surgery and repair or resection and anastomosis done. One patient died due to haemorrhagic complications and rest recovered uneventfully.
\end{abstract}

KEY WORDS: JEJUNUM; DIVERTICULA; COMPLICATION; PERFORATION

SHORT TITLE: Perforations of jejunal diverticula

HOW TO CITE: Ray J, Singh PK, Kumar S, Sahu SK, Sachan PK. Perforations complicating jejunal diverticula: our experience. Jurnalul de chirurgie (Iaşi). 2013; 9(1): 85-88. DOI: 10.7438/1584-9341-9-1-11.

\section{INTRODUCTION}

Diverticula of intestine is a pouch or pocket extending through the layers of the bowel wall. Diverticula is of true type when it contains all layers of intestinal wall. A false diverticula lacks the muscle layer. Jejunal diverticula is the rarest of small intestinal diverticula. Jejunal diverticula are rare and single ones moreover.

Incidence has been reported as 0.5 to $2.3 \%$ from radiological study and $0.3 \%$ to $4.5 \%$ from autopsies. Many of the diverticula remain silent or may be responsible for non-specific chronic abdominal symptoms such as abdominal pain, nausea, flatulence, and diarrhea. Jejunal diverticula may be complicated by perforation, bleeding, obstruction [1-3].

In this retrospective study we present three cases of single jejunal diverticula complicated by perforation and peritonitis.

\section{CASE REPORTS}

Case 1

The patient was a 65 year obese old man with the complaint of abdominal pain, constipation and fever for last 3 days. Physical examination revealed a tender and rigid abdomen with masked liver dullness and an empty rectum. X-ray abdomen revealed free gas under both domes of the diaphragm and ground glass opacity of rest of abdomen.

Haemogram revealed haemoglobin $12 \mathrm{~g} / \mathrm{dL}$, total leucocyte count $17,000 / \mathrm{mm}^{3}$. Ultrasonogram revealed distended bowel loops and free fluid in the peritoneal cavity. Urgent exploratory laparotomy was done and a perforated single jejunal diverticula was found about 1 foot from duodenojejunal flexure.

The diverticula was in the mesenteric side of jejunum. 
Peritoneal cavity was filled with purulent fluid and bile. The segment of jejunum was resected and end-to-end anastomosis was done along with meticulous peritoneal lavage and drainage. A feeding jejunostomy was constructed distal to the anastomosis. Patient developed pneumonia in post-operative period but eventually improved without any further morbidity.

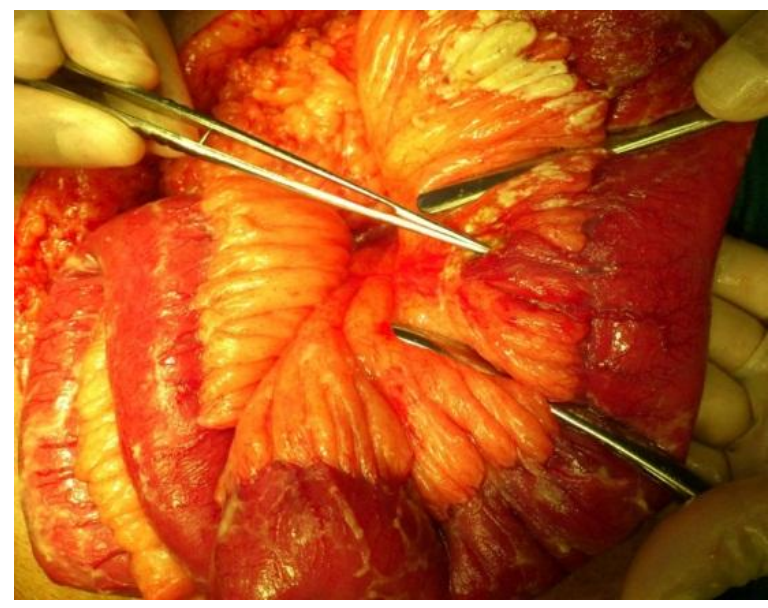

Fig. 1 Case 1: intraoperative photograph showing perforated mesenteric jejunal diverticula

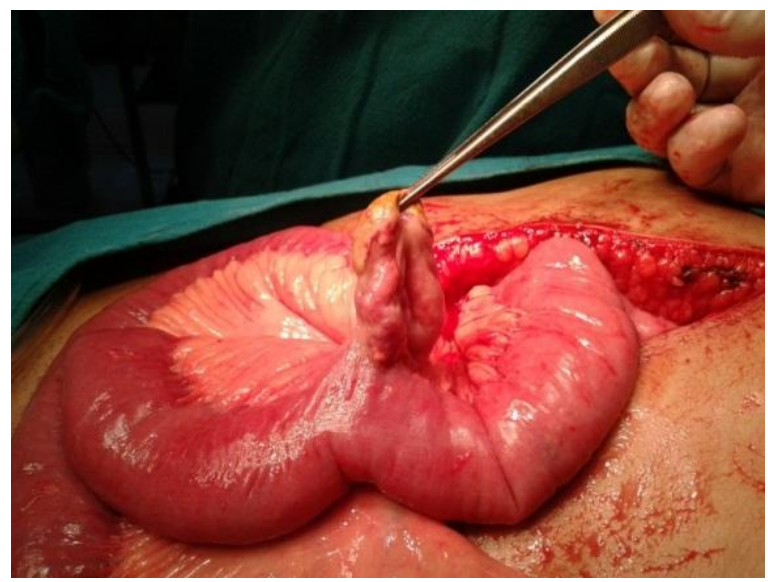

Fig. 2 Case 2: intraoperative photograph showing perforated mesenteric jejunal diverticula

\section{Case 2}

The 48 year old patient was being treated for haemorrhagic dengue when he suddenly developed severe pain in the upper abdomen and subsequent constipation and distension of the abdomen. His abdomen was tender, tense and rigid. Rebound tenderness was present. X-ray demonstrated free gas under the domes of diaphragm. His haemoglobin was $9 \mathrm{gm} / \mathrm{dL}$, total leucocyte count was $11,000 / \mathrm{mm}^{3}$ and total platelet count was $40,000 / \mathrm{mm}^{3}$.

Patient was taken for emergency exploratory laparotomy. A single perforated jejunal diverticula was found about 2 feet from duodenojejunal flexure. It was leaking contents abundantly. The diverticula was narrow mouthed and midway between mesenteric and antimesenteric border. The perforation was at the apex and the base was evidently not involved.

The diverticula was resected at its base and primary repair of jejunum was done. Patient was managed postoperatively in intensive care unit and succumbed to hemorrhagic dengue.

\section{Case 3}

This 53 year old male patient was admitted to the emergency with complaints of abdominal pain, constipation, and distension of abdomen for 2 days. Physical examination demonstrated a distended, tender and rigid abdomen, masked liver dullness and empty rectum. X-ray showed free gas under diaphragm and features of generalized peritonitis. Patient's haemoglobin was $11 \mathrm{~g} / \mathrm{dL}$, total leucocyte count was $14,600 / \mathrm{mm}^{3}$, and blood urea nitrogen was $56 \mathrm{mg} / \mathrm{dL}$.

Emergency exploratory laparotomy was done. A single jejunal diverticula perforated at the apex was found about one and a half foot away from duodenojejunal flexure. It was situated in the antimesenteric aspect of jejunum. Wedge resection of the diverticula and adjacent jejunal wall was done with primary repair in two layers. Feeding jejunostomy was created for postoperative enteral feeding. Patient recovered uneventfully.

\section{DISCUSSION}

Diverticula of the gastrointestinal tract are fairly common and most are of colonic type. True diverticulas contain all layers of the bowel wall and false ones are simply herniation of bowel mucosa through its wall. False diverticulas are mostly acquired. They probably occur because of motor 
dysfunction of the smooth muscle or the myenteric plexus of the small intestine that generate increased intraluminal pressures (jejuno-ileal dyskinesia) and peristalsis .The mucosa herniates usually in the areas where mesenteric vessels pierce bowel wall. Therefore the majority is situated on the mesenteric side, but diverticula on antimesenteric side are also found which are rarer. Jejunal diverticula often coexist with colonic ones [4].

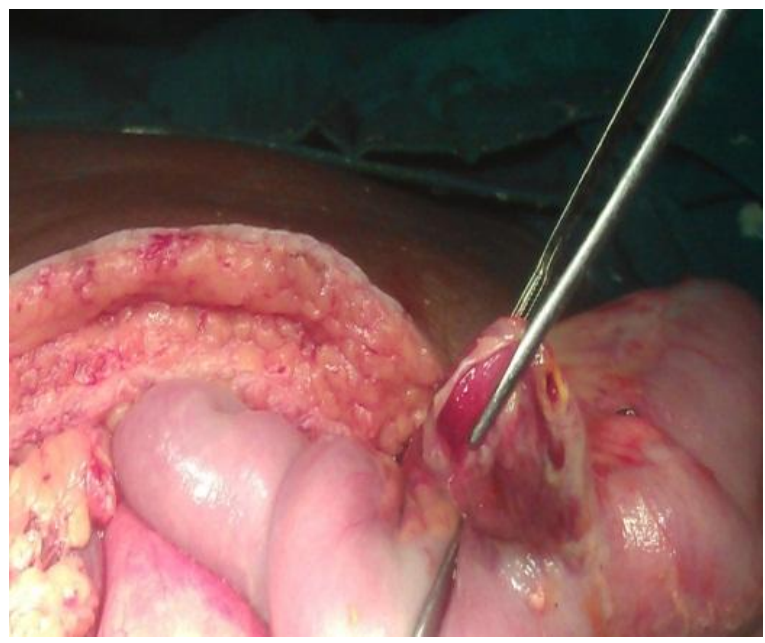

Fig. 3 Case 3: perforated solitary antimesenteric jejunal diverticula

Multiple jejunal diverticulas are rare and single diverticulas are rarer. These are found more commonly in the elderly and a slight male preponderance has been noted. Mickley found an incidence of $4 \%$ among patients aged more than 70 years [5].

Jejunal diverticula are mostly asymptomatic, may give rise to symptoms like pain, flatulence, and borborygmi, may produce malabsorption syndrome. It may also be complicated by hemorrhage, perforation, obstruction necessitating surgery in $10 \%$ of cases [6].

Jejunal diverticula may present as perforation due to necrotising inflammation $(82 \%)$, trauma $(12 \%)$ or foreign body impaction (6\%). Eventually generalised peritonitis or localised intraperitoneal abscess may form. Clinical features are extremely variable, so the imaging modalities are essential in reaching diagnosis.
Symptoms vary from vague intermittent abdominal pain highly variable in location, to acute abdominal pain, with associated leucocytosis. But these can also be encountered during emergency laparotomies for hollow viscus perforation implied by clinical feature and free gas under the diaphragm as with our three cases. CT scan is the best imaging modality in these cases. CT typically shows nonspecific changes that are consistent with inflammation and infection. Frequent descriptions include evidence of an inflammatory mass containing gas, wall thickening of the involved segment, and oedema of the surrounding tissues, including fat or fascial planes [7].

Management of perforation is by resecting the involved segment with primary anastomosis. The surgical treatment options are segmental jejunal resection and end-toend anastomosis. But this is possible if the perforated diverticula are sufficiently away from DJ junction.

The surgical options become limited in perforations very close to DJ junction, as there is hardly any length of jejunal available for joining together. Moreover majority of patients are elderly with co-morbidity in perforated duodenal diverticula, the peritonitis is usually in retroperitoneal plane and conservative approach is adopted. If at all operation is decided, it involves complete diversion of gastric and biliary flow from affected area of duodenum. The surgical option may be closure of mouth of diverticula with a purse string suture, wedge excision of the diverticula or formal resection and anastomosis of the segment. Previous studies have shown that a laparoscopic approach is successful. Extensive resection should be avoided as this has the potential to lead to short bowel syndrome $[8,9]$.

Novak et al. have demonstrated a few cases where a localised perforation of jejunal diverticula could be treated non-surgically with either intravenous antibiotic therapy or computed tomography-assisted percutaneous drainage of the abscess [10]. 
Bleeding is another important complication of jejunal diverticula. It usually presents as lower gastrointestinal bleed but rare cases of haematemesis have been reported. These can be diagnosed by CT scan or small bowel contrast studies, but technetium red cell tagged scan and mesenteric angiography are more specific and offer scope of arterial embolization.

In haemodynamically unstable patients need urgent laparotomy and resection of bleeding segment and primary anastomosis [11].

Intestinal obstruction may complicate jejunal diverticula. Large diverticula may cause intussusception. Enterolith and gallstones may cause mechanical obstruction and dyskinesia cause non-mechanical type of obstruction. Diagnosis is aided by X-ray and CT scan. Whatever the cause may be, the management is similar as with obstruction of other parts of small bowel. During laparotomy the enteroliths may be crushed and milked back into the colon [12].

\section{CONCLUSIONS}

Jejunal diverticulas are rare and may present with a variety of clinical spectrum. Awareness about their complications, early diagnosis and prompt intervention may prevent life threatening complications.

\section{CONFLICT OF INTERESTS}

None to declare

\section{REFERENCES}

1. Margolies NM. Diverticular disease of the small bowel. In: Morris JP, Wood CW, editors. Oxford Textbook of Surgery. New York: Oxford University Press; 2000. p. 13751380.

2. Wilcox RD, Shatney CH. Surgical implications of jejunal diverticula. South Med J. 1988; 81(11): 1386-1391.

3. Fisher JK, Fortin D. Partial small bowel obstruction secondary to ileal diverticulitis. Radiology. 1977; 122(2): 321-322.

4. Ahmedi SK, Saeed MH. Antimesenteric jejunal diverticulosis: A case report. Eur $J$ Surg Sci. 2010; 1(3): 96-98.

5. Mickley V, Reismann B. The prevalence and significance of pseudodiverticulosis of the small intestines. Dtsch Med Wochenschr. 1989; 114(33): 1237-1241.

6. Chugay P, Choi J, Dong XD, Palder SB, Frey CB. Jejunal diverticular disease complicated by enteroliths: Report of two different presentations. World J Gastrointest Surg. 2010; 2(1): 26-29.

7. Graña L, Pedraja I, Mendez R, Rodríguez R. Jejuno-ileal diverticulitis with localized perforation: CT and US findings. Eur J Radiol. 2009; 71(2): 318-323.

8. Surov A, Stock K. Jejunal diverticulosis. Eur J Med Res. 2005; 10(8): 358-360.

9. Miller G, Mueller C, Yim D, Macari M, Liang H, Marcus S, Shamamian P. Perforated Duodenal diverticulitis: report of three cases. Dig Surg. 2005; 22(3): 198-202.

10. Novak JS, Tobias J, Barkin JS. Nonsurgical management of acute jejunal diverticulitis: a review. Am J Gastroenterol 1997; 92: 19291931.

11. Vilallonga R, Sanchez Garcia JL, Armengol $\mathrm{M}$, Iordache N. Jejunal diverticula causing massive intestinal bleeding. Chirurgia. 2012; 107(5): 652-654.

12. Steenvoorde P, Schaardenburgh P, Viersma JH. Enterolith ileus as a complication of jejunal diverticulosis: two case reports and a review of the literature. Dig Surg. 2003; 20(1): 57-60. 